\title{
Traumatismos dentários: conhecimento dos cirurgiões dentistas da atenção básica à
} saúde

\author{
Dental traumatisms: primary health care dentists' knowledge
}

\section{Traumatismos dentales: conocimiento de los cirujanos dentistas de la atención básica a} la salud

\section{Recebido: 28/07/2017 \\ Aprovado: 17/12/2017 \\ Publicado: 05/04/2018}

Carlus Alberto Oliveira dos Santos ${ }^{1}$ Aparecida Tharlla Leite de Caldas ${ }^{2}$ Ricardo Liberalino Ferreira de Souza ${ }^{3}$ Mafalda Siewes ${ }^{4}$ Margarida Maria Pontes de Carvalho ${ }^{5}$ Fernanda de Araújo Trigueiro Campos ${ }^{6}$

Esta pesquisa tem como objetivo avaliar o conhecimento de Cirurgiões Dentistas (CD) da Atenção Básica à Saúde de João Pessoa - PB sobre traumatismo dentário através de um questionário validado por Pedrini em 2008. A amostra foi composta por 70 CD classificados em 2 grupos: G1 (CDs que concluíram a graduação há um tempo menor ou igual a 25 anos) e G2 (CDs que concluíram a graduação há mais de 25 anos). Aplicou-se os testes QuiQuadrado de Pearson e/ou o teste Exato de Fischer no software IBM SPSS (20.0). As respostas obtidas foram heterogêneas em relação ao trauma mais prevalente no serviço. Os CD responderam que não sabiam tratar: consussão (52,9\%), luxações extrusivas (88,7\%), luxações laterais (90,0\%), luxações intrusivas $(75,0 \%)$ e 75,9\% responderam não saber tratar todos os tipos de traumatismo. Verificou-se diferença estatisticamente significativa entre o tempo de conclusão do curso de graduação e a autopercepção de que seria capaz de tratar todos os tipos de traumatismos $(\mathrm{p}<0,05)$. Concluiu-se que os CDs avaliados não apresentam conhecimento satisfatório para tratar os traumatismos dentários.

Descritores: Traumatismos dentários; Saúde pública; Qualidade de vida.

This research aims to evaluate the knowledge of dental surgeons (DS) in the primary health care of João PessoaPB about dental injuries through a questionnaire evaluated by Pedrini in 2008. The sample was made up of 70 DSs, which were classified in 2 groups: G1 (DSs that finished their graduation 25 years ago or less) and G2 (DSs which finished their graduation more than 25 years ago). Pearson's Chi-squared test and/or Fisher's exact test were used in the IBM software SPSS (20.0). The answers of the participants were varied regarding the most common trauma in their practice. The DSs stated not to know how to treat: concussions (52.9\%), extrusive luxations (88.7\%), lateral luxations (90.0\%), intrusive luxations (75.0\%), and 75.9\% said not to know how to treat any type of injury. Statistically significant differences were found between the time since the participants concluded their graduation courses and the self-perception they have on the types of injury they are capable of treating ( $p>0.05)$. The evaluated DSs did not show adequate knowledge to deal with dental trauma.

Descriptors: Tooth injurie; Public health; Quality of life.

Esta investigación tiene como objetivo evaluar el conocimiento de Cirujanos Dentistas (CDs) de la Atención Básica a la salud de João Pessoa - PB (Paraíba) sobre traumatismo dental a través de un cuestionario validado por Pedrini en 2008. La muestra estaba compuesta por 70 CDs clasificados en 2 grupos: G1 (CDs que concluyeron la graduación hace 25 años o menos) y G2 (CDs que concluyeron la graduación hace más de 25 años). Se aplicaron los tests chiCuadrado de Pearson y/o test Exacto de Fischer en el software IBM SPSS (20.0). Las respuestas obtenidas fueron heterogéneas en relación al trauma más frecuente en el servicio. Los CDs respondieron que no sabían tratar: concusión (52,9\%), luxaciones extrusivas (88,7\%), luxaciones laterales (90\%), luxaciones intrusivas (75\%) y 75,9\% respondieron no saber tratar todos los tipos de traumatismo. Se verificó diferencia estadísticamente significativa entre el tiempo de conclusión de la carrera de graduación y la autopercepción de que sería capaz de tratar todos los tipos de traumatismos $(\mathrm{p}<0,05)$. Se concluye que los CDs evaluados no presentan conocimiento satisfactorio para tratar los traumatismos dentales.

Descriptores: Traumatismos de los dientes; Salud pública; Calidad de vida.

\footnotetext{
${ }^{1}$ Graduando em Odontologia pelo Centro Universitário de João Pessoa (UNIPÊ), João Pessoa, PB, Brasil. 0RCID: 0000-0002-5988-1186 E-mail: carlusodonto@gmail.com

${ }^{2}$ Graduanda em Odontologia pelo UNIPÊ, João Pessoa, PB, Brasil. ORCID: 0000-0002-2720-5853. E-mail: cida.tcharlla@gmail.com

${ }^{3}$ Graduando em Odontologia pelo UNIPÊ, João Pessoa, PB, Brasil. ORCID: 0000-0003-4858-7034. E-mail: ricardo.liberalino@hotmail.com

${ }^{4}$ Cirurgiã Dentista. João Pessoa, PB, Brasil. ORCID: 0000-0002-2152-1724 E-mail: claudiomafalda@bol.com.br

${ }^{5}$ Cirurgiã Dentista. Mestre e Doutora em Odontopediatria. Professora Titular da Faculdade Integrada de Patos - FIP, Patos, PB, Brasil. ORCID: 0000-0001-95520-9983 E-mail: margotpontes@hotmail.com

${ }^{6}$ Cirurgiã Dentista. Doutora em Odontopediatria. Professora Assistente do Curso de Odontologia da UNIPÊ, João Pessoa, PB, Brasil. ORCID: 0000-0002-5590-3085 E-mail: fe_trigueiro@hotmail.com
} 


\section{INTRODUÇÃO}

$\mathrm{O}$ traumatismo dentário (TD) pode ser definido como uma injúria de gravidade, intensidade e extensão variável, podendo ser de origem acidental ou intencional. Esses eventos adquirem um caráter especial quando comparados com os principais problemas que influenciam direta ou indiretamente a qualidade de vida. Juntamente com a cárie e o câncer de boca, o traumatismo de acometimento dentário encontra-se entre os principais agravos de saúde pública mundial1-3.

A negligência em relação ao tratamento odontológico após o TD pode ter como consequência: alteração de cor, mobilidade, alteração de posição na arcada dentária, sintomatologia dolorosa, sensibilidade, reabsorções radiculares ou ósseas, necrose e perda do elemento dental. O TD não é causa de doenças, mas pode ser responsável por problemas que vão além dos estéticos. Os danos ocasionados pelos variados tipos de injúrias dentais envolvem o bem-estar psicológico, funcional e social; além de produzir custos significativos para as vítimas ${ }^{2,3}$.

No caso das crianças, esses danos podem afetar seu comportamento e progresso escolar, além de influenciar na qualidade de vida de seus pais ou cuidadores. As crianças são mais suscetíveis às injúrias dentais, e os elementos dentários mais afetados são os incisivos centrais superiores, o que se deve ao fato de que as crianças se envolvem ativamente com atividades ao ar livre, prática bastante comum nas zonas rurais da Paraíba ${ }^{4}$.

Dentre os variados fatores associados à etiologia do traumatismo dentário, tem-se: quedas, atividades esportivas, acidentes automobilísticos e violência, além de fatores anatômicos que favorecem a ocorrência de traumas dentais, tais como: sobressaliência e sobremordida $^{1-3}$.

Por se tratar de um problema de saúde pública, é necessária a participação de uma equipe multidisciplinar na atenção às crianças vítimas de traumatismos dentários. Educadores físicos, professores, cuidadores de creches e agentes comunitários de saúde são exemplos desses profissionais, que na maioria das vezes, estão presentes de forma ativa no dia a dia das crianças ${ }^{1,3}$.

$\mathrm{Na}$ Estratégia Saúde da Família, particularmente, a equipe de saúde bucal encontra-se em situação especial, por possuir acesso à população no consultório, no domicílio, na escola e na comunidade, no sentido da realização de treinamentos de familiares, professores e demais profissionais da saúde para esta situação emergencial em Odontologia ${ }^{3,5}$.

Diante desse contexto é relevante que os Cirurgiões Dentistas tenham conhecimento técnico adequado e continuado sobre as variadas formas de TD, contribuindo para a melhoria da qualidade de vida da população ${ }^{5}$.

Esta pesquisa tem como objetivo avaliar o conhecimento de Cirurgiões Dentistas (CD) da Atenção Básica à Saúde de João Pessoa - PB sobre o traumatismo dentário, através de um questionário validado por Pedrini em $2008^{6}$.

\section{MÉTODO}

Trata-se de um estudo de caráter exploratório, quantitativo e descritivo. A coleta de dados foi realizada nas Unidades de Saúde da Atenção Básica na cidade de João Pessoa-PB, com os Cirurgiões Dentistas (CD) inscritos regularmente no Conselho Regional de Odontologia (CRO).

A pesquisa iniciou-se após aprovação do Comitê de Ética em Pesquisa do UNIPÊ, através da certidão CAAE: 50595415.8.0000.5176.

0 universo desse estudo foi constituído por $180 \mathrm{CD}$ regularmente inscritos no Conselho Regional de Odontologia, com qualquer especialidade, em qualquer faixa etária de ambos os gêneros inscritos no CRO, sessão Paraíba.

Para a realização do cálculo amostral, considerou-se um grau de confiança de 95\% e erro de 5\%, resultando uma amostra com 123 Cirurgiões Dentistas. Considerando que ocorreram perdas, dessa amostra de 123 CDs restaram apenas 70. As perdas se justificam da seguinte forma: os CDs não quiseram responder ao questionário, estavam de férias, ou encontravam-se de licença médica.

A pesquisa ocorreu mediante a leitura e assinatura do Termo de Consentimento Livre 
e Esclarecido. Foram excluídos da pesquisa CDs que não faziam parte das Unidades Básicas de Saúde (UBS).

0 instrumento de coleta de dados tratase de um questionário previamente validado ${ }^{6}$. Esse questionário contém 9 questões e visa identificar o conhecimento dos CDs sobre traumatismos dentários.

Os questionários foram entregues aos participantes que aceitaram participar da pesquisa. As perguntas continham somente uma alternativa como resposta, devendo ser indicada a assertiva de maior importância. 0 participante teve o tempo que julgou necessário para responder ao questionário, que ao término foi guardado em um envelope, preservando sua identificação.

Os dados foram analisados mediante estatística descritiva, que correspondeu ao cálculo de frequências absolutas e percentuais das respostas dos questionários aplicados aos CDs.

Os participantes da pesquisa foram classificados em 2 grupos de acordo com o ano de conclusão da graduação: G1 (CDs que concluíram a graduação há um tempo menor ou igual a 25 anos) e G2 (CDs que concluíram a graduação há mais de 25 anos). As respostas relacionadas às condutas clínicas foram dicotomizadas em adequadas e inadequadas.

Empregou-se a análise de diferença de proporção (teste qui-quadrado de Pearson e/ou teste exato de Fischer) para determinar eventuais diferenças entre os grupos de profissionais e a avaliação das condutas instituidas para cada tipo de traumatismo. 0 nível de significância foi fixado em $\mathrm{p}<0,05$. Todas as análises foram realizadas usando o software IBM SPSS versão 20.0 e considerando um intervalo de confiança de 95\%.

\section{RESULTADOS}

Após análise dos dados observou-se que, dentre os pesquisados, mais da metade $(82,9 \%)$ eram do sexo feminino, acima de 30 anos, com menos de 25 anos de formado $(61,4 \%)$, e com formação predominante numa universidade pública federal (82,9\%), conforme resultados da Tabela 1 .

Os dados observados na Tabela 2 indicam os profissionais com especialização ou não, e a área da mesma. Observa-se que a maior parte dos participantes, representados pela frequência de $82,9 \%$, tem especialização, sendo Prótese (8,9\%), Endodontia $(7,4 \%)$ e Periodontia $(5,4 \%)$ os destaques.

A Tabela 3 apresenta os traumatismos mais frequentes nos serviços de atuação dos CDs pesquisados.

A Tabela 4 mostra o índice de acertos e erros dos CDs avaliados. Na grande maioria das perguntas predominaram erros, exceto para subluxação $(60,8 \%$ de acertos $)$. A maioria dos participantes diz não se sentir preparado para a intervir como um todo $(79,5 \%)$. 
Tabela 1. Profissionais de acordo com as características sociodemográficas, ano de conclusão da graduação e instituição onde concluiu a graduação. João Pessoa, 2016.

\begin{tabular}{lcc}
\hline Variáveis & $\mathbf{n}$ & $\mathbf{\%}$ \\
\hline Sexo & 58 & 82,9 \\
Feminino & 12 & 17,1 \\
Masculino & & \\
Faixa etária & 6 & 8,8 \\
$\leq 29$ anos & 9 & 13,2 \\
$30-39$ anos & 12 & 17,6 \\
$40-49$ anos & 25 & 36,8 \\
$50-59$ anos & 16 & 23,5 \\
$\geq 60$ anos & & \\
Ano de conclusão da graduação & 43 & 61,4 \\
$\leq 25$ anos & 27 & 38,6 \\
$>25$ anos & & \\
Instituição onde concluiu a graduação & 58 & 82,9 \\
UFPB & 3 & 4,3 \\
UNIPÊ & 3 & 4,3 \\
UEPB & 1 & 1,4 \\
UFRN & 1 & 1,4 \\
FORNE & 1 & 1,4 \\
UNP & 1 & 1,4 \\
UPE & 1 & 1,4 \\
UNB & 1 & 1,4 \\
URNE & &
\end{tabular}

Tabela 2. Profissionais de acordo a especialização, ano de conclusão da especialização, e relato de atuação em outras áreas. João Pessoa, 2016.

\begin{tabular}{|c|c|c|}
\hline Variáveis & $\mathbf{N}$ & $\%$ \\
\hline \multicolumn{3}{|c|}{ Possui curso de especialização? } \\
\hline Sim & 58 & 82,9 \\
\hline Não & 12 & 17,1 \\
\hline \multicolumn{3}{|l|}{ Se sim, qual? } \\
\hline Prótese & 5 & 8,9 \\
\hline Endodontia & 4 & 7,1 \\
\hline Periodontia & 3 & 5,4 \\
\hline Implantodontia & 2 & 3,6 \\
\hline Ortodontia & 2 & 3,6 \\
\hline Dentística & 1 & 1,8 \\
\hline Cirurgia & 1 & 1,8 \\
\hline Odontologia Legal & 1 & 1,8 \\
\hline Odontopediatria & 1 & 1,8 \\
\hline Odontologia do Trabalho & 1 & 1,8 \\
\hline Outros & 35 & 62,5 \\
\hline \multicolumn{3}{|l|}{$\begin{array}{l}\text { Ano de conclusão da } \\
\text { especialização }\end{array}$} \\
\hline$\leq 19$ anos & 5 & 11,4 \\
\hline$>19$ anos & 37 & 84,1 \\
\hline Em andamento & 2 & 4,5 \\
\hline \multicolumn{3}{|c|}{$\begin{array}{l}\text { Além da especialização, atuação } \\
\text { em outras áreas }\end{array}$} \\
\hline Sim & 42 & 70,0 \\
\hline Não & 18 & 30,0 \\
\hline \multicolumn{3}{|l|}{ Áreas } \\
\hline Clínica Geral & 20 & 36,4 \\
\hline Prótese & 3 & 5,5 \\
\hline Dentística & 2 & 3,6 \\
\hline Periodontia & 1 & 1,8 \\
\hline Mais de uma & 29 & 52,7 \\
\hline
\end{tabular}


Tabela 3. Profissionais conforme relato de trauma mais prevalente no serviço de atuação e a avaliação das condutas instituídas para cada tipo de traumatismo. João Pessoa, 2016.

\begin{tabular}{lcc}
\hline Variáveis & N & \% \\
\hline Trauma mais prevalente no & & \\
serviço de atuação & 15 & 23,4 \\
\hline Avulsão & 7 & 10,9 \\
\hline Luxação lateral & 3 & 4,7 \\
Concussão & 3 & 4,7 \\
Luxação intrusiva & 3 & 4,7 \\
Intrusão & 3 & 4,7 \\
Luxação extrusiva & 2 & 3,1 \\
Subluxação & 28 & 43,8 \\
Mais de um & & \\
Conduta para Concussão & 24 & 47,1 \\
Adequada & 27 & 52,9 \\
Inadequada & & \\
Conduta para Subluxação & 31 & 60,8 \\
$\quad$ Adequada & 20 & 39,2 \\
Inadequada & & \\
Conduta para Luxação & & 11,3 \\
Extrusiva & 6 & 88,7 \\
Adequada & 47 & 10,0 \\
Inadequada & & 90,0 \\
Conduta para Luxação Lateral & 5 & \\
Adequada & 45 & 25,0 \\
Inadequada & & 75,0 \\
Conduta para Luxação Intrusiva & 13 & \\
Adequada & 39 & 20,5 \\
Inadequada & & 79,5 \\
Capaz de tratar os tipos de & 8 & \\
traumatismos apresentados & 31 & \\
Sim & & \\
Não & & \\
\hline
\end{tabular}

Tabela 4. Profissionais de acordo com o tempo de conclusão da graduação e avaliação das condutas instituídas para cada tipo de traumatismo. João Pessoa, 2016.

\begin{tabular}{|c|c|c|c|c|c|c|c|}
\hline \multirow[b]{3}{*}{ Variáveis } & \multicolumn{6}{|c|}{ Tempo de conclusão da graduação } & \multirow{3}{*}{ p-valor } \\
\hline & \multicolumn{2}{|c|}{$\leq 25$ anos } & \multicolumn{2}{|c|}{$>25$ anos } & \multicolumn{2}{|c|}{ Total } & \\
\hline & $\mathbf{N}$ & $\%$ & $\mathbf{n}$ & $\%$ & $\mathbf{N}$ & $\%$ & \\
\hline & \multicolumn{4}{|c|}{ Conduta para Concussão } & & & $0,069^{*}$ \\
\hline Adequada & 9 & 34,6 & 15 & 60,0 & 24 & 47,1 & \\
\hline \multirow[t]{2}{*}{ Inadequada } & 17 & 65,4 & 10 & 40,0 & 27 & 52,9 & \\
\hline & \multicolumn{4}{|c|}{ Conduta para Subluxação } & & & $0,645^{*}$ \\
\hline Adequada & 15 & 57,7 & 16 & 64,0 & 31 & 60,8 & \\
\hline \multirow[t]{2}{*}{ Inadequada } & 11 & 42,3 & 9 & 36,0 & 20 & 39,2 & \\
\hline & \multicolumn{6}{|c|}{ Conduta para Luxação Extrusiva } & $0,999 * *$ \\
\hline Adequada & 3 & 10,7 & 3 & 12,0 & 6 & 11,3 & \\
\hline \multirow[t]{2}{*}{ Inadequada } & 25 & 89,3 & 22 & 88,0 & 47 & 88,7 & \\
\hline & \multicolumn{6}{|c|}{ Conduta para Luxação Lateral } & $0,999 * *$ \\
\hline Adequada & 2 & 8,0 & 3 & 12,0 & 5 & 10,0 & \\
\hline \multirow[t]{2}{*}{ Inadequada } & 23 & 92,0 & 22 & 88,0 & 45 & 90,0 & \\
\hline & \multicolumn{6}{|c|}{ Conduta para Luxação Intrusiva } & $0,262^{*}$ \\
\hline Adequada & 5 & 18,5 & 8 & 32,0 & 13 & 25,0 & \\
\hline Inadequada & 22 & 81,5 & 17 & 68,0 & 39 & 75,0 & \\
\hline \multicolumn{7}{|c|}{ Você seria capaz de tratar todos os tipos de traumatismo apresentados? } & $0,020 * *$ \\
\hline Sim & 1 & 5,0 & 7 & 36,8 & 8 & 20,5 & \\
\hline Não & 19 & 95,0 & 12 & 63,2 & 31 & 79,5 & \\
\hline
\end{tabular}




\section{DISCUSSÃO}

No presente estudo, os participantes do sexo feminino foram mais prevalentes, conforme resultados descritos, corroborando um estudo ${ }^{1}$ realizado anteriomente que avaliou o impacto do traumatismo dentário na qualidade de vida de crianças e adolescentes.

Em pesquisa ${ }^{4}$ realizada com Cirurgiões Dentistas frente ao tratamento emergencial de pacientes com avulsão dentária em Alfenas-MG, verificou-se que 58,3\% eram do sexo masculino, 8,3\% tinham entre 51 a 60 anos de idade, $11,1 \%$ com até 30 anos de formados e 38,9\%.

No presente estudo a maioria dos participantes afirmou ter alguma especialização (82,9\%), dos quais a maior parte $(84,1 \%)$ concluiu a especialização há mais de 19 anos. Azevedo ${ }^{9}$ encontrou resultados semelhantes em seu estudo, no qual $56 \%$ dos 182 profissionais relataram ter alguma especialização. Outro estudo ${ }^{4}$ relatou que dos 72 profissionais pesquisados (86,1\%), eram pós-graduados.

Os resultados desse trabalho divergem de outra investigação ${ }^{7}$, na qual a necrose pulpar foi relatada como a sequela mais comumente encontrada, desenvolvendo-se no primeiro ano após o traumatismo. A calcificação pulpar foi citada como a sequela mais comumente encontrada após fraturas radiculares. Anquilose e reabsorção radicular por substituição foram encontradas após avulsão.

Torna-se necessário o conhecimento de que as sequelas relatadas podem aparecer após os traumatismos, para que o Cirurgião Dentista esteja apto a reconhecer os sintomas e sinais clínicos e radiográficos, fornecendo um prognóstico e traçando um plano de tratamento.

Nesse estudo observou-se que as respostas foram heterogêneas em relação ao trauma mais prevalente no serviço de atuação. Em relação às condutas adotadas frente a diferentes tipos de trauma, a maior parte dos profissionais não soube responder quais são as condutas adequadas para tratar concussões, bem como luxações extrusivas, laterais e intrusivas.
Questionados se seriam capazes de tratar todos os tipos de traumatismo apresentados, a maioria respondeu que não. Semelhantemente, em outro estudo $6,74,8 \%$ de 469 profissionais tiveram condutas inadequadas para tratar subluxação, sendo $95,3 \%$ para luxações extrusivas, e $88,9 \%$ para laterais. Nas condutas para tratar concussão, os resultados do presente estudo divergem dos de Pedrini ${ }^{6}$, onde $26,5 \%$ dos profissionais tiveram condutas adequadas.

Outra pesquisa ${ }^{8}$ semelhante discorda dos resultados do presente estudo quando relata que foram analisados 129 prontuários de pacientes com traumatismo dentoalveolar, e as condutas adotadas variaram, sendo 44 casos $(34,1 \%)$ encaminhados ao projeto da Universidade Estadual de Maringá, que possibilita a centralização do atendimento clínico a pacientes que sofreram traumatismo dental.

Nesta pesquisa 79,5\% dos profissionais relataram que não seriam capazes de tratar todos os tipos de traumatismos apresentados. 0 manejo adequado do trauma dentoalveolar inclui um correto diagnóstico inicial, tratamento de urgência imediato se necessário, tratamento definitivo e a preservação do caso ${ }^{2}$.

De acordo com o presente estudo, quanto maior o tempo de formação, maior a capacidade relatada para tratar todos os tipos de traumatismo. Esses achados divergem dos resultados encontrados por Pedrini 6 , que em um estudo realizado com 469 profissionais observou que, os Cirurgiões Dentistas formados há menos tempo conseguiram elaborar maior quantidade de planos adequados, principalmente para o traumatismo de concussão, o que provavelmente se deve à abordagem mais recente sobre o assunto.

Resultados diferentes do presente estudo foram relatados por outro trabalho ${ }^{5}$, realizado no Programa de Saúde da Família de Campina Grande/PB em 2009. Nele, os profissionais encontravam-se em situação especial, por possuírem acesso à população no consultório, no domicílio, na escola e na comunidade, no sentido da realização do treinamento de familiares, professores e 
demais profissionais da saúde para esta situação emergencial em Odontologia.

É de fundamental importância que os Cirurgiões Dentistas tenham conhecimento técnico adequado e continuado sobre o assunto em tela, contribuindo para melhoria da qualidade de vida da população.

\section{CONCLUSÃO}

A partir dos resultados desse estudo, é possível concluir que os Cirurgiões Dentistas não apresentam conhecimento suficiente para tratar de maneira adequada as injúrias dentárias.

Por sua vez, o conhecimento dos Cirurgiões Dentistas relacionado aos traumatismos dentário é menor com o passar dos anos de formado.

\section{REFERÊNCIAS}

1. Antunes DP, Gonçalves MA, Antunes DP, Paula MVQ, Leite FPP, Miranda MDGA. Conhecimento dos cirurgiões-dentistas sobre avulsão dentária. UNOPAR Cient Ciênc Biol Saúde. 2013;15(1):5-8.

2. Francisco SS, Souza Filho FJ, Pinheiro ET, Murrer $\mathrm{RD}$, Jesus AS. Prevalence of traumatic dental injuries and associated factors among Brazilian school children. Oral Health Prev Dent. 2013; 11(1):31-8. 3. Souza BLMD, Lopes PHDS, Nogueira EFDC, Torres BCA. Manejo de trauma dentoalveolar atípico: relato de caso. Rev Cir Traumatol Buco-Maxilo-Fac. 2013; 13(4):45-50.

4. Lima DC, Pereira AA, Swerts AA, Fernandes LA. Conduta dos cirurgiões dentistas de Alfenas/MG frente ao tratamento emergencial de pacientes com avulsão dentária. Arq Odontol. 2013; 49(4):169-76.
5. Antunes LAA, Leao A, Maia TLC. Impacto do traumatismo dentário na qualidade de vida de crianças e adolescentes: revisão crítica e instrumentos de medida. Ciênc Saúde Coletiva. 2012; 17(12):3417-24.

6. Pedrini D. Análise do conhecimento dos cirurgiões dentistas sobre plano de tratamento das injúrias do ligamento periodontal após traumatismo dento alveolar. [Tese]. Araçatuba, SP: UNESP; 2008. 117f. 7. Morello J, Ribeiro FC, Roldi A, Pereira RS, Barroso JM, Intra JBG. Sequelas subsequentes aos traumatismos dentários com envolvimento endodôntico. Rev Bras Pesqui Saúde. 2011; 13(2):68-73.

8. Tolentino LS, Camarini ET, Tolentino ES, Iwaki Filho L, Endo MS, Pavan AJ. Traumatismo dentoalveolar: análise dos casos atendidos no serviço de residência em cirurgia e traumatologia bucomaxilofacial da Universidade Estadual de Maringá no período de 2004 a 2006. Rev Odontol UNESP. 2008; 37(1):53-57.

9. Azevedo RA. Avaliação do conhecimento dos cirurgiões dentistas sobre as condutas adotadas frente a traumatismos alveolodentários. [Dissertação]. Pelotas, RS: UFPEL; 2012. 57f.

CONTRIBUIÇÕES
Carlus Alberto Oliveira Santos e
Aparecida Tharlla Leite Caldas
realizaram a interpretação dos dados e
redação. Mafalda Siewes foi responsável
pela concepção, delineamento, análise e
interpretação dos dados. Ricardo
Liberalino Ferreira de Souza, Fernanda
de Araújo Trigueiro Campos e Margarida
Maria Pontes de Carvalho atuaram na
concepção, delineamento, análise e
interpretação dos dados e, revisão crítica.

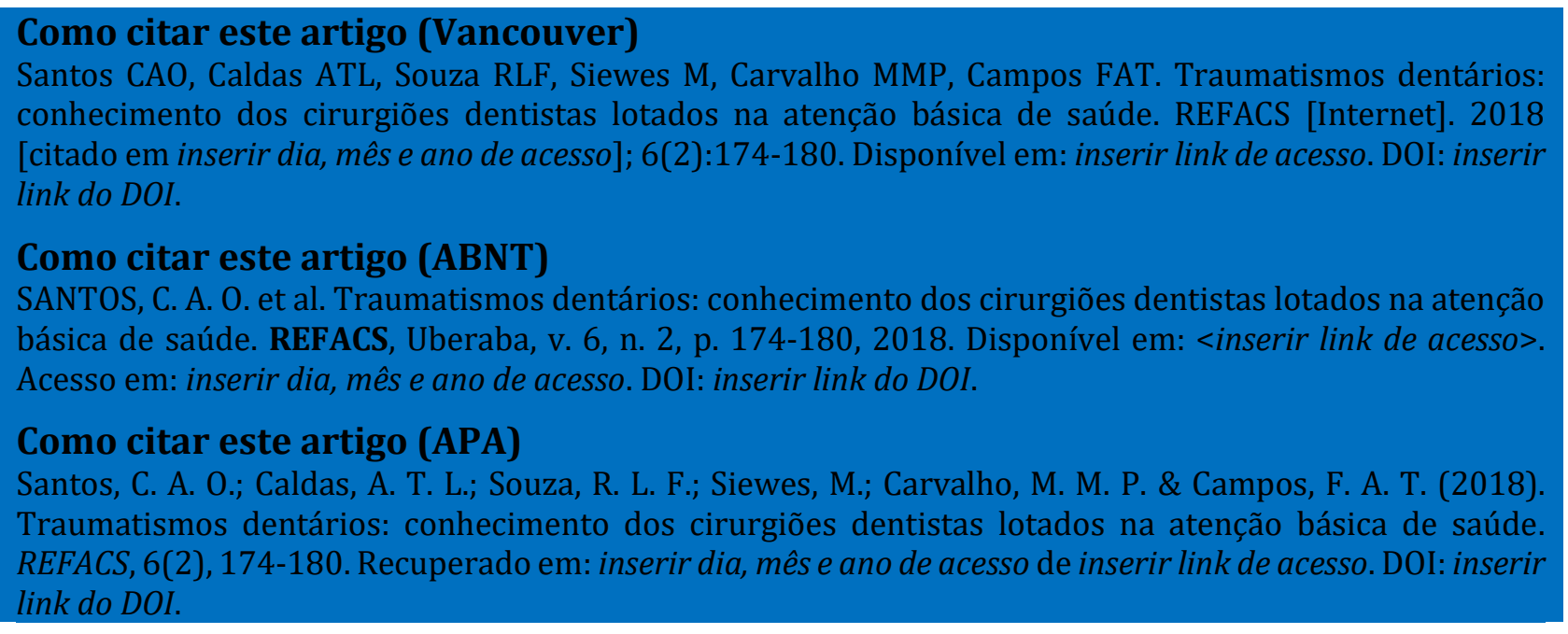

